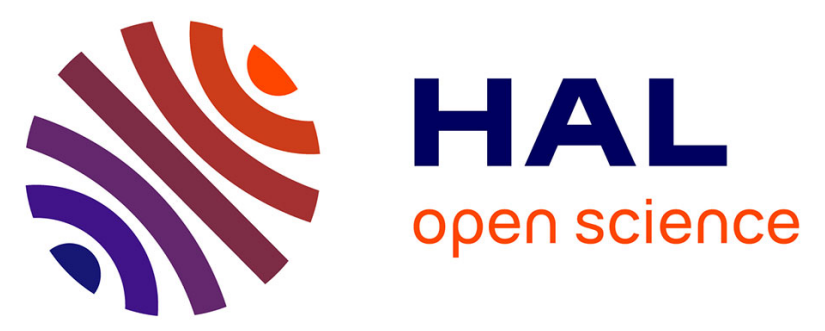

\title{
Influence of Strong Confinement on the Structure and Dynamics of Liquids: a Study of the Clay/Water Interface Exploiting 2 H NMR Spectroscopy and Spin-Locking Relaxometry
}

Patrice Porion, Anne-Marie Faugère, Anne-Laure Rollet, Emmanuelle Dubois, Virginie Marry, Laurent Michot, Alfred Delville

\section{To cite this version:}

Patrice Porion, Anne-Marie Faugère, Anne-Laure Rollet, Emmanuelle Dubois, Virginie Marry, et al.. Influence of Strong Confinement on the Structure and Dynamics of Liquids: a Study of the Clay/Water Interface Exploiting $2 \mathrm{H}$ NMR Spectroscopy and Spin-Locking Relaxometry. Journal of Physical Chemistry C, 2018, 122 (29), pp.16830-16841. 10.1021/acs.jpcc.8b05089 . hal-02383136

\section{HAL Id: hal-02383136 https://hal.science/hal-02383136}

Submitted on 10 Dec 2021

HAL is a multi-disciplinary open access archive for the deposit and dissemination of scientific research documents, whether they are published or not. The documents may come from teaching and research institutions in France or abroad, or from public or private research centers.
L'archive ouverte pluridisciplinaire HAL, est destinée au dépôt et à la diffusion de documents scientifiques de niveau recherche, publiés ou non, émanant des établissements d'enseignement et de recherche français ou étrangers, des laboratoires publics ou privés. 
This document is confidential and is proprietary to the American Chemical Society and its authors. Do not copy or disclose without written permission. If you have received this item in error, notify the sender and delete all copies.

\section{Influence of Strong Confinement on the Structure and Dynamics of Liquids: A Study of the Clay/Water Interface Exploiting ${ }^{2} \mathrm{H}$ NMR Spectroscopy and Spin-Locking Relaxometry}

\begin{tabular}{|r|l|}
\hline Journal: & The Journal of Physical Chemistry \\
\hline Manuscript ID & jp-2018-050893.R1 \\
\hline Manuscript Type: & Article \\
\hline Date Submitted by the Author: & n/a \\
\hline Complete List of Authors: & $\begin{array}{l}\text { Porion, Patrice; CNRS-Universite d'Orleans, Interfaces, Confinement, } \\
\text { Materiaux et Nanostructures (ICMN) } \\
\text { Faugère, Anne Marie; CNRS-Universite d'Orleans, Centre de Recherche sur } \\
\text { la Matiere Div. } \\
\text { Rollet, Anne-Laure; cnrs, PECSA } \\
\text { Dubois, Emmanuelle; Université Pierre et Marie Curie, Laboratoire PHENIX } \\
\text { Marry, Virginie; UPMC, } \\
\text { Michot, Laurent; PhENix(Physicochimie des Electrolytes et Nanosystèmes } \\
\text { Interfaciaux), UMR 8234 - CNRS - UPMC } \\
\text { Delville, Alfred; CNRS, CRMD }\end{array}$ \\
\hline
\end{tabular}

\section{SCHOLARONE" \\ Manuscripts}


p. 1

\title{
Influence of Strong Confinement on the Structure and Dynamics of Liquids: A Study of the Clay/Water Interface Exploiting ${ }^{2} \mathrm{H}$ NMR Spectroscopy and Spin-Locking Relaxometry
}

\author{
Patrice Porion $^{\mathrm{a},{ }^{*}}$, Anne Marie Faugère ${ }^{\mathrm{a}}$, Anne-Laure Rollet $^{\mathrm{b}}$, Emmanuelle Dubois $^{\mathrm{b}}$, \\ Virginie Marry ${ }^{\mathrm{b}}$, Laurent J. Michot ${ }^{\mathrm{b}}$, and Alfred Delville ${ }^{\mathrm{a},{ }^{*}}$ \\ ${ }^{\text {a } I n t e r f a c e s, ~ C o n f i n e m e n t, ~ M a t e r i a u x ~ e t ~ N a n o s t r u c t u r e s, ~ I C M N, ~ U M R ~ 7374, ~}$ \\ CNRS - Université d'Orléans, 45071 Orléans Cedex 02, France \\ ${ }^{\mathrm{b}}$ Physicochimie des Electrolytes et Nanosystèmes Interfaciaux, PHENIX, UMR 8234, \\ CNRS - Sorbonne Université, 4 place Jussieu, 75252 Paris Cedex 5, France
}

\section{*Corresponding authors:}

E-mail: delville@cnrs-orleans.fr (A.D.) and porion@cnrs-orleans.fr (P.P.). 


\begin{abstract}
${ }^{2} \mathrm{H}$ NMR spectroscopy, multi-quanta relaxation and spin-locking relaxometry are used to investigate the structural and dynamical properties of water molecules confined within dense sediments of synthetic fluoro-hectorite. As shown by the large residual splitting of the ${ }^{2} \mathrm{H}$ NMR resonance line, water molecules confined in the interlamellar space of the clay are strongly oriented at contact with the fluorinated basal surface of the clay. Multi-quanta relaxation measurements are used to identify and quantify the contributions of the quadrupolar and hetero-nuclear dipolar couplings monitoring the NMR relaxation of the confined water molecules. Finally, the average residence time of the water molecules confined within the interlamellar space of the clay platelets is quantified by a detailed analysis of ${ }^{2} \mathrm{H}$ spin-locking relaxometry measurements. Thanks to the significant contributions of both quadripolar and hetero-nuclear dipolar relaxation mechanisms, ${ }^{2} \mathrm{H}$ spin-locking relaxation measurements probe a broad dynamical range, by sampling angular velocities ranging between $10^{2}$ and $3 \times 10^{5} \mathrm{rad} / \mathrm{s}$.
\end{abstract}




\section{Introduction}

In the last decades, solid/liquid interfaces were the subject of numerous experimental $^{1,2}$ and theoretical ${ }^{3,4}$ studies since confinement greatly modifies the structural and dynamical properties ${ }^{5-13}$ of fluids. In that context, clay/water interfacial systems were frequently investigated for two reasons. Firstly, from a theoretical point of view, clay platelets are ideal models for studying solid/liquid interfaces since they have an atomically smooth surface with well-characterized structure and atomic composition. Secondly, natural and synthetic clays are employed in many industrial applications (drilling, waste storing, ionic exchange, heterogeneous catalysis) exploiting their various physico-chemical properties ${ }^{14}$ (swelling, thixotropy, large specific surface area and surface charge density, adsorbing power, surface acidity). Monitoring and predicting the mobility of confined molecules in the porous network of clay aggregates is thus of prime importance for optimizing a large class of such industrial applications. As a consequence, numerous experimental studies have been performed to determine the mobility of confined fluids over a broad range of diffusing time. At short time-scale (between $10^{-12}$ and $10^{-7}$ seconds), Inelastic ${ }^{15-18}$ and Quasi-Elastic ${ }^{13,19,20}$ Neutron Scattering were frequently used to obtain information on the local mobility of the confined fluids. By contrast, the long-time mobility (above milli-seconds) of fluids is generally probed by using pulsed gradient spin echo (PGSE) NMR spectroscopy ${ }^{21,22}$. But under confinement, the large enhancement of the NMR relaxation rates ${ }^{23,24}$ generally prohibits the use of PGSE NMR spectroscopy. Recent ${ }^{2} \mathrm{H}$ NMR relaxations measurements ${ }^{25}$ have illustrated the large enhancement of deuterium NMR relaxation rate of heavy water confined within dense clay sediments. In that context, NMR relaxometry ${ }^{26}$ provides an interesting alternative way to obtain information on the long-time mobility of confined fluids. For that purpose, we performed ${ }^{2} \mathrm{H}$ spin-locking relaxometry measurements, because a broad frequency domain may be probed by quadrupolar nuclei (with spin $I>1 / 2$ ) under 
confinement $^{26-30}$. By contrast with spin-locking NMR relaxometry ${ }^{26-30}$, both PGSE-NMR spectroscopy $^{21,22}$ and field-cycling NMR relaxometry ${ }^{22,31}$ require incompressible time-delays necessary either to create stable magnetic field gradients, in the case of PGSE, or switch the strength of the static magnetic field, in the case of field-cycling relaxometry. These inherent time-delays strongly limit the applicability of these experimental procedures for investigating the dynamical behavior of strongly confined fluids because of the enhancement of their NMR relaxation rates induced by confinement ${ }^{23,24}$.

In that study we selected to use a synthetic clay sample (fluoro-hectorite) in order to avoid the tremendous enhancement of the NMR relaxation induced by paramagnetic impurities generally present in natural clay ${ }^{26,27}$. Furthermore, substitution of the structural hydroxides at the clay surface by fluorine atoms greatly simplifies the interpretation of ${ }^{2} \mathrm{H}$ NMR spectra since all the deuterium atoms detected by ${ }^{2} \mathrm{H}$ NMR spectroscopy pertain to the added water molecules. The ${ }^{2} \mathrm{H}$ NMR spectra are first recorded to quantify the degree of ordering of confined water molecules. The influence of surface hydrophobicity on the organization of confined water molecules is clearly demonstrated by comparing the measured residual quadrupolar splitting to values previously reported, under the same experimental conditions, for classical hectorite with hydroxides in the clay layer. Multi-quanta ${ }^{2} \mathrm{H}$ relaxation measurements are performed for various orientations of the clay film by reference with the static magnetic field $\mathbf{B}_{\mathbf{0}}$ in order to quantify the contributions of the quadrupolar and heteronuclear dipolar couplings responsible for the relaxation of confined water molecules. Finally, spin-locking relaxometry measurements are performed to determine the frequency variation of the NMR relaxation rates. This dynamical information gives direct access to the average residence time of the water molecules confined in the interlamellar space of clay platelets. Such analysis of the ${ }^{2} \mathrm{H}$ NMR relaxation measurements requires a complete analysis 
of the time evolution of the components of the spin=1 nuclei under the influence of the NMR relaxation mechanisms in addition to the residual quadrupolar splitting and the irradiation pulses.

\section{Materials and Methods}

\section{II.1. Sample Preparation}

Figure 1

The clay sample used in that study is a synthetic hectorite with the general formula $\mathrm{Si}_{8}\left(\mathrm{Mg}_{5.2} \mathrm{Li}_{0.8}\right) \mathrm{O}_{20} \mathrm{~F}_{4} \mathrm{Cs}_{0.8}$. It results from the sandwiching of one octahedral layer of magnesium oxides between two tetrahedral layers of silica ${ }^{14}$. The hydroxide atoms generally located within the hexagonal cavity of the two silica layers are replaced by fluorine atoms, strongly modifying the water affinity for the clay surface ${ }^{32,33}$. The negative charge of the clay platelets results from substitutions, in the octahedral layer, of some magnesium by lithium. These negative charges are neutralized by cesium cations localized in the interlamellar space between clay platelets. As determined by transmission electronic microscope $\mathrm{e}^{30}$, the average size of the individual clay platelet is $(0.3 \pm 0.1) \mu \mathrm{m}$. More details on the synthesis and characterization of that clay sample are given in the literature ${ }^{34}$. Oriented clay membranes (10 $\mu \mathrm{m}$ thickness) were prepared by sedimentation of a dilute dispersion $(\sim 20 \mathrm{~g} / \mathrm{L})$ and airdried on a flat surface. Successive deposits were stacked to form a macroscopic film with a $3 \mathrm{~mm}$ thickness ${ }^{30}$. As a consequence, the macroscopic clay film exhibits multi-scale structure since it results from the superposition of numerous individual membranes each composed from partially oriented clay aggregates (see Figure 1). As determined by X-ray diffraction ${ }^{32}$, the thickness of these clay aggregates roughly corresponds to 20 clay platelets. The macroscopic clay film was equilibrated with bulk heavy water at a relative humidity close 
to $97 \%$, leading to the formation of one hydration layer ${ }^{33}$. As measured by water adsorption/desorption isotherms ${ }^{30}$, the total water content of this $\mathrm{Cs}^{+} /$fluorohectorite sample is $3.9 \mathrm{mmol}$ of water per gram of dry clay, corresponding roughly to four water molecules per cesium cation. Obviously, the first hydration layer of cesium cation immersed in bulk water contains more than four water molecules ${ }^{35}$. In our case, strong confinement results from the formation of a single hydration layer ${ }^{33}$. As a consequence, ions and water molecules are located in the equatorial plane at the center of the interlamellar space between the clay platelets, thus restricting hydration of the confined counterions. A fragment of the clay film (20 $\mathrm{mm}$ length, $3 \mathrm{~mm}$ width) is cut and inserted in a glass tube fitting the inner cavity of the homemade NMR detection coil. To avoid clay desiccation during NMR experiments, a droplet of heavy water is deposed in the sealing cap of the glass tube, i.e. at a position as far as possible from the NMR detection coil.

\section{II.2. NMR Measurements}

Figure 2

${ }^{2} \mathrm{H}$ NMR spectra and relaxation measurements of confined heavy water were recorded on a DSX360 Bruker spectrometer operating at a static magnetic field $\mathbf{B}_{0}$ of $8.465 \mathrm{~T}$. On this spectrometer, typical pulse duration for the total inversion of the longitudinal magnetization is equal to $9 \mu$ s by using a homemade detection coil. Spectra were recorded using a fast acquisition mode with spectral width of $1 \mathrm{MHz}$ with a numerical spectral resolution of $125 \mathrm{~Hz}$. The spectra and relaxation measurements were performed at various orientations of the clay film as described by the $\beta^{L F}$ Euler angle between the direction of the static magnetic field $\mathbf{B}_{0}$ and the macroscopic film director $\vec{n}^{F}$ (see Figure 2). 


\section{II.3. Numerical simulations}

The LAMMPS package ${ }^{38}$ (http://lammps.sandia.gov) was used to perform numerical simulations of Molecular Dynamics (MD) of the clay/water interface by exploiting the classical CLAYFF force field ${ }^{39}$ adapted to fluoro-hectorite ${ }^{20}$. The interactions between water molecules are described by using the TIP4P/2005 model of bulk water ${ }^{40}$. The general formula of the fluoro-hectorite unit cell is $\mathrm{Cs}_{0.8} \mathrm{Si}_{8}\left(\mathrm{Mg}_{5.2} \mathrm{Li}_{0.8}\right) \mathrm{O}_{20} \mathrm{~F}_{4}$. The simulation cell contains two clay lamellae, each composed from 40 unit cells. The number of confined water molecules (4 $\mathrm{H}_{2} \mathrm{O}$ per Cs cation) was selected to reproduce the water uptake of fluoro-hectorite ${ }^{30}$ neutralized by $\mathrm{Cs}^{+}$cations. The interlamellar separation between the two clay lamellae is also fixed at $12.4 \AA^{32}$. MD simulations were performed in the NVT ensemble to evaluate the trajectories during $5 \mathrm{~ns}$. The water mobility in the direction parallel to the clay surface is evaluated from the asymptotic slope of the corresponding mean squared displacements. 
p. 8

\section{Results and Discussion}

\section{III.1. $\quad{ }^{2} H$ NMR Spectra}

Figure 3

As displayed in Figure $3,{ }^{2} \mathrm{H}$ NMR spectra of the hydrated clay sediment exhibit the coexistence of two spin populations characterized by a broad doublet and a thin central resonance line corresponding to confined and bulk water, respectively. As previously detected for clay sediments ${ }^{25-29}$, the quadrupolar splitting $v_{Q}^{o b s}$ of confined water molecules results from specific orientation of their $O D$ director by reference to the director, noted $\vec{n}^{F}$, pertaining to their confining clay platelets, in agreement with results obtained by numerical simulations $^{25-29}$. As a consequence, the detected quadrupolar splitting $v_{Q}^{\text {obs }}$ varies as a function of the orientation $\beta^{L F}$, into the static magnetic field $\mathbf{B}_{0}$, of the macroscopic clay film according to the relationship (see the SI):

$$
v_{Q}^{o b s}\left(\beta^{L F}\right)=\left|\frac{3 \cos ^{2}\left(\beta^{L F}\right)-1}{2}\right| \times\left\langle\frac{3 \cos ^{2}\left(\beta^{F D}\right)-1}{2}\right\rangle \times 210 \mathrm{kHz}
$$

where the first second order Legendre polynomial $P_{2}\left(\cos \left(\beta^{L F}\right)\right)=0.5\left(3 \cos ^{2}\left(\beta^{L F}\right)-1\right)$ describes the orientation of the macroscopic clay film into the static magnetic field characterized by the $\beta^{L F}$ Euler angle (see Section II.2) and the second Legendre polynomial $P_{2}\left(\cos \left(\beta^{F D}\right)\right)$, into the bracket, describes the average orientation of the $O D$ directors of the confined water molecules defined by their $\beta^{F D}$ Euler angle by reference with the normal to their confining clay platelets (see the SI). Finally, the quadrupolar coupling constant (QCC) of deuterium $\left({ }^{2} \mathrm{H}\right)$ in heavy water ${ }^{41}$ is equal to $210 \mathrm{kHz}$. In addition to confined water, bulk water 
molecules are localized either in the cap of the glass tube (see Section II.1) or within the micro-porosity between the aggregates of clay platelets. By contrast with confined water molecules, bulk water molecules are randomly oriented and exchange slowly, at the NMR time-scale ${ }^{42}$, with the confined water molecules leading to a thin central resonance line fully independent of the film orientation within the static magnetic field $\mathbf{B}_{0}$. By contrast with fieldcycling NMR relaxometry, the stability and homogeneity of the static magnetic field induced by the supraconducting magnet (see Section II.2) lead to a experimental spectral resolution of the ${ }^{2} \mathrm{H}$ NMR spectra of the order of a few $100 \mathrm{~Hz}$, i.e. largely sufficient to distinguish the contributions of the confined and bulk water molecules to the total detected magnetization (see Figure 2).

As displayed in Figure 3, the maximum residual splitting $(57 \pm 3) \mathrm{kHz}$ detected for a parallel alignment between the film director $\vec{n}^{F}$ and the static magnetic field $\mathbf{B}_{0}$ is much larger than values previously measured at room temperature for heavy water confined within a large class of clays ${ }^{25-29,41}$. One may be tempted to attribute this surprisingly large splitting to the exchange between deuterium atoms of confined heavy water and protons from the clay structure. Such interpretation is not plausible since hydroxylates on the basal surface of the clay platelet are totally replaced by fluorine atoms (see Section II.1). Furthermore, the total number of hydroxylates localized on the edge surface of clay platelets is two orders of magnitude smaller than the number of deuterium atoms pertaining to confined water molecules. As a consequence, the large residual splitting reported here is the fingerprint of a strong organization of confined water molecules since the corresponding order parameter (i.e. the averaged Legendre polynomial of Eq. 2) must be equal to $(0.36 \pm 0.02)$. By contrast, previous ${ }^{2} \mathrm{H}$ NMR measurements lead to much smaller order parameter $(0.10 \pm 0.01)$ for water molecules confined within hydroxylated hectorite neutralized by sodium counterions 
under the similar hydration conditions ${ }^{28,41}$. By contrast, the order parameter of water molecules confined within the same hydroxylated hectorite increases slightly to 0.15 when sodium counterions are replaced by cesium ${ }^{41}$. As a consequence, the high value reported here for the order parameter of confined water molecules is not induced by the chemical nature of the neutralizing monovalent cation but illustrates the large influence of the fluorine atoms located at the center of the ditrigonal cavity on the organization of the water molecules confined within the interlamellar space between the clay platelets.

The residual order parameter evaluated by MD simulations, $\left\langle P_{2}\left(\cos \left(\beta^{F D}\right)\right)\right\rangle \approx 0.204$, is significantly smaller than the experimental value, restricting the validity of the potentials used here to describe the hydration of fluoro-hectorite neutralized by Cesium counterions. Despite the large deficiency of the model, the numerical data may nevertheless be used in some qualitative way to illustrate the organization of the confined water molecules. As displayed in Figure $4 \mathrm{a}$, the oxygen atoms of the water molecules are predominantly localized in the equatorial plane of the clay interface. Three peaks are easily identified in the concentration profile of the hydrogen atoms, suggesting a specific orientation of the confined water molecule characterized by one $O D$ director pointing along the normal to the clay platelets (noted $\vec{n}^{F}$ ). Figure $4 \mathrm{~b}$ validates that analysis by exhibiting a bimodal distribution of the $O D$ directors by reference to $\vec{n}^{F}$ : in addition to a broad distribution around $70^{\circ}$ $\left\{69.5^{\circ}=\operatorname{acos}(0.35)\right\}$, an intense sharp peak appears at $0^{\circ}$ corresponding to water molecules pointing one $O D$ director parallel to $\vec{n}^{F}$ the normal to the clay platelet. Further information on the water orientation in the interlamellar space are given by the distributions of the directors describing the water dipole and the normal to the $D O D$ plane of the water molecules by reference to $\vec{n}^{F}$. As illustrated by their broad distribution in Figure $4 \mathrm{~b}$, the directors perpendicular to the $D O D$ plane are not totally perpendicular to the normal $\vec{n}^{F}$ to 
the clay platelet. As a consequence, the orientation of the water dipole by reference to the normal $\vec{n}^{F}$ exhibits a broad distribution around $60^{\circ}\left\{58.7^{\circ}=\operatorname{acos}(0.52)\right\}$ while an angle of $52.26^{\circ}$ should occur for water molecules with an $O D$ director perfectly pointing to the clay surface with its $D O D$ plane perpendicular to the clay surface. The degree of ordering of the confined water molecule is further illustrated in Figure 5, where the white vertical and horizontal lines correspond to the orientation of the perfectly oriented water molecule mentioned above.

Figures $4 \mathrm{a}$ and $4 \mathrm{~b}$

Figure 5

\section{III.2. ${ }^{2}$ H Multi-Quanta NMR Relaxation Measurements}

Figure 6

The relaxation of the $T_{10}^{I R}$ coherence (also called longitudinal relaxation rate $R_{1} \equiv R_{10}$ ) of bulk water present in the sample was measured by using a classical saturation-recovery pulse sequence ${ }^{43}$. The average longitudinal relaxation rate measured here for bulk water $\left(R_{10}=25 \pm 5 \mathrm{~s}^{-1}\right)$ is one order of magnitude larger than the longitudinal relaxation rate previously reported for pure $\mathrm{D}_{2} \mathrm{O}\left(R_{10}=2.5 \pm 0.1 \mathrm{~s}^{-1}\right)^{44}$. In order to selectively measure the longitudinal relaxation rate of confined water molecules we used a saturation-recovery pulse sequence followed by a double-quanta filtering (see Figure 6a). As displayed in Figure 6a, the duration of the first detection pulse was selected to optimize the transfer of magnetization between the $T_{10}^{I R}$ and $T_{22}^{I R}(a)$ coherences, see Eq. S5d in the SI. In the same manner, the duration of the second detection pulse optimizes the $T_{22}^{I R}(a)$ to $T_{11}^{I R}(s)$ transfer of coherence 
(see Eq. A5b in the SI). As previously reported for other clay/water interfaces ${ }^{25-29}$, the longitudinal relaxation rate of confined water molecules $\left(R_{10}=130 \pm 30 \mathrm{~s}^{-1}\right)$ is significantly enhanced by reference with the relaxation rate of pure water $\left(R_{10}=2.5 \pm 0.1 \mathrm{~s}^{-1}\right)^{44}$.

By contrast with bulk liquids, the relaxation rates of liquids confined within $2 \mathrm{D}$ interfacial systems vary as a function of the orientation of the interface by reference with the direction of the static magnetic field $\mathbf{B}_{\mathbf{0}}{ }^{25-29}$. As a consequence, it becomes crucial to extract from the angular variation of the apparent relaxation rates the contributions of the various relaxation mechanisms to the intrinsic relaxation rates evaluated in the frame of the confining lamellae. This may be performed by using the Wigner rotation matrices ${ }^{45}$ that relates the apparent spectral densities $J_{m}^{X, a p p}(\omega)$ measured in the laboratory frame to the intrinsic spectral density $J_{m}^{X, \text { int }}(\omega)$ evaluated in the frame attached to the clay film, where $X$ stems for the relaxation mechanisms $X \in\{Q, D\}$ and $m \in\{0,1,2\}$, see Eqs S9 and S12 in the SI:

$$
\begin{aligned}
& J_{0}^{X, a p p}\left(\beta^{L F}, \omega\right)=\frac{1}{4}\left(1-3 \cos ^{2} \beta^{L F}\right)^{2} J_{0}^{X, \text { int }}(\omega)+\frac{3}{4}\left(\sin 2 \beta^{L F}\right)^{2} J_{1}^{X, \text { int }}(\omega) \\
& +\frac{3}{4}\left(\sin \beta^{L F}\right)^{4} J_{2}^{X, i n t}(\omega) \\
& J_{1}^{X, a p p}\left(\beta^{L F}, \omega\right)=\frac{3}{2} \cos ^{2} \beta^{L F} \sin ^{2} \beta^{L F} J_{0}^{X, \text { int }}(\omega)+\frac{1}{2}\left(1-3 \cos ^{2} \beta^{L F}+4 \cos ^{4} \beta^{L F}\right) J_{1}^{X, \text { int }}(\omega) \\
& +\frac{1}{2}\left(1-\cos ^{4} \beta^{L F}\right) J_{2}^{X, \text { int }}(\omega) \\
& J_{2}^{X, a p p}\left(\beta^{L F}, \omega\right)=\frac{3}{8}\left(1-\cos ^{2} \beta^{L F}\right)^{2} J_{0}^{X, \text { int }}(\omega)+\frac{1}{2}\left(1-\cos ^{4} \beta^{L F}\right) J_{1}^{X, \text { int }}(\omega) \\
& +\frac{1}{8}\left(1+6 \cos ^{2} \beta^{L F}+\cos ^{4} \beta^{L F}\right) J_{2}^{X, \text { intrinsic }}(\omega)
\end{aligned}
$$


The relaxation of the $T_{21}^{I R}(a)$ and $T_{22}^{I R}(a)$ coherences are thus measured as a function of the orientation of the macroscopic clay film into the static magnetic field $\mathbf{B}_{0}$ probed by the Euler angle $\beta^{L F}$, in order to extract the relative contributions of the quadrupolar and heteronuclear dipolar relaxation mechanisms (see the SI). These relaxation measurements are again performed by using an initial saturation pulses train followed by multi-quanta filtering (see Figure 6b-c). As detailed in Eqs S6b-c in the SI, the angular velocities $k_{2}$ and $k_{3}$, monitoring the time evolution of the various coherences (see Eqs.S5b-d in the SI) vary as a function of the residual quadrupolar coupling $\omega_{Q}$. Because of the large residual quadrupolar coupling detected here, the durations of the filtering pulses are thus specifically selected for each orientation of the clay sample according to the set of Eqs S5b-d (see the SI) in order to optimize the coherence transfers implied in these relaxation measurements. These settings of the pulse durations may reasonably neglect the contribution from the NMR relaxation mechanisms to the time evolution of the coherences since the pulse durations (typically a few $\mu \mathrm{s}$ ) are short enough compared to the NMR relaxation times. In order to remove the contribution from the central resonance line, our analysis focuses on the frequency domains covered by the satellite. Figures 7 and 8 illustrate typical spectra measured during the time evolution of $T_{21}^{I R}(a)$ and $T_{22}^{I R}(a)$ coherences for an orientation of the macroscopic film director either parallel $\left(\beta^{L F}=0^{\circ}\right)$ or perpendicular $\left(\beta^{L F}=90^{\circ}\right)$ to the static magnetic field $\mathbf{B}_{0}$, respectively.

Figure 7 and Figure 8

During the time evolution of the $T_{21}^{I R}(a)$ coherence (see Figure 7), we expected to detect a single resonance line occurring at the characteristic residual quadrupolar splitting 
given by Eq. 1 (i.e. $57000 \mathrm{~Hz}$ at $\beta^{L F}=0^{\circ}$ and $27000 \mathrm{~Hz}$ at $\beta^{L F}=90^{\circ}$ ). By contrast, the stacked spectra displayed in Figure 7 clearly exhibit curved resonance bands whose number increases as a function of the time delay. By using the set of Eqs S8 and S11 in the SI, we simulate the time evolution of the various coherences during each elementary step of the pulse sequence displayed in Figure 6b. It then becomes possible to reproduce the structure of the stacked spectra (see Figure 7) by including in our numerical model a Gaussian distribution of the orientation of the individual clay directors by reference to the director of the macroscopic film. The same approach was used to simultaneously analyze the time evolution of the $T_{22}^{I R}(a)$ coherence (see Figure 8) measured by the pulse sequence displayed in Figure 6c.

The whole set of experimental data displayed in Figures 7 and 8 is fairly well reproduced by using a limited number of parameters quantifying the maximum residual splitting corresponding to a parallel orientation of the clay film $v_{Q}^{\max }=(57 \pm 2) \mathrm{kHz}$, the intrinsic contributions of the quadrupolar (see Eq 2a with $X=Q): J_{0}^{Q, \text { int }}(0)=(550 \pm 50) \mathrm{s}^{-1}$, $J_{1}^{Q, \text { int }}(0)=(5000 \pm 1000) \mathrm{s}^{-1}$ and $J_{2}^{Q, \text { int }}(0)=(120 \pm 20) \mathrm{s}^{-1}$, and dipolar (see Eq 2a with $X=D): J_{0}^{D, \text { int }}(0)=J_{1}^{D, \text { int }}(0)=(2700 \pm 300) \mathrm{s}^{-1}$ and $J_{2}^{D, \text { int }}(0)=(1700 \pm 300) \mathrm{s}^{-1}$ relaxation mechanisms, in addition to the width of the Gaussian distribution of the individual clay directors $\vec{n}^{F}$ in the macroscopic film with $\sigma_{\beta}=(17 \pm 3)^{\circ}$. By contrast with bulk liquids, both sets of intrinsic contributions to the quadrupolar $J_{m}^{Q, \text { int }}(0)$ with $m \in\{0,1,2\}$ and heteronuclear dipolar $J_{m}^{D, \text { int }}(0)$ with $m \in\{0,1,2\}$ relaxation mechanisms vary as a function of the index $m$, leading to angular dependent transverse relaxation rates (see Eqs 2a). That behavior is induced by the water confinement within the clay interlamellar space, breaking down the isotropy of the distribution of the Euler angles $\left(\theta^{L W}, \phi^{L W}\right)$ characterizing the orientation of the 
$O D$ directors of the water molecules into the static magnetic field $\mathbf{B}_{0}$. As a consequence, in the case of confined water molecules the average values of the second order spherical harmonics $Y_{2, m}\left(\theta^{L W}, \phi^{L W}\right)$ with $m \in\{0,1,2\}$ implied in the derivation of the quadripolar and dipolar Hamiltonians (see the SI) differ significantly. In that context, the significant enhancement of the $J_{1}^{Q, \text { int }}(0)$ contribution induces a corresponding increase of the transverse relaxation rate for orientations of the film director close to $\beta^{L F}=45^{\circ}$ by reference with the static magnetic field $\mathbf{B}_{0}$. That specific angle corresponds indeed to the maximum value of the $\left(\sin 2 \beta^{L F}\right)^{2}$ function weighting the contribution of $J_{1}^{Q, \text { int }}(0)$ in the derivation of the apparent transverse relaxation rate (see Eq. 2a). In the same manner, the smallest transverse relaxation rate is always detected for a perpendicular orientation of the film director (i.e. $\beta^{L F}=90^{\circ}$ ) by reference with the direction of the static magnetic field $\mathbf{B}_{0}{ }^{25-29}$.

\section{III.3. ${ }^{2}$ H NMR Spin-Locking Relaxometry}

Figure 9

As a consequence, spin-locking relaxation measurements were performed for a perpendicular orientation of the film director, i.e. $\beta^{L F}=90^{\circ}$, in order to optimize the signal/noise ratio of the doublet describing the dynamical properties of confined water molecules. Figure 9 illustrates the pulse sequences used for these spin-locking relaxation measurements: after a first excitation pulse, the irradiation power is applied for variable evolution time before acquisition of the transverse magnetization. In the so-called $T_{2 \rho}$ spinlocking relaxation measurement, both excitation and irradiation pulses have the same phase ${ }^{46}$ while for $T_{1 \rho}$ spin-locking relaxation measurement, they are shifted by $90^{\circ 47}$. The spin-locking measurements were performed at five different irradiation powers characterized by 
corresponding angular velocities $\omega_{1}$ of ${ }^{2} \mathrm{H}$ nuclei $\left(\right.$ i.e. $8.7 \times 10^{4}, 5.1 \times 10^{4}, 2.0 \times 10^{4}, 1.3 \times 10^{4}$, and $\left.0.63 \times 10^{4} \mathrm{rad} \cdot \mathrm{s}^{-1}\right)$. As explained in the SI, under the simultaneous influence of irradiation power $\omega_{1}$ and residual static quadrupolar coupling $\left(\omega_{Q}\right)$, three different angular velocities, noted $k_{1}, k_{2}$ and $k_{3}$, monitor the time evolution of the coherences of spin $I=1$ nuclei (see Eqs S5-6 in the SI). Figure 10 illustrates the range of angular velocities that can be potentially probed by our spin-locking relaxation measurements. As explained in the SI, the first eigen-mode (i.e. $k_{1}$ ) is probed thanks to the contribution of the quadrupolar relaxation mechanism (see Eq. S4a in the SI), covering here a limited dynamical range (see Figure 10). Hopefully, the next two eigen-modes (i.e. $k_{2}$ and $k_{3}$ ) are probed by the hetero-nuclear dipolar relaxation mechanism (see Eq. S5a in the SI) extending by three decades the probed dynamical range (see Figure 10) while the initial range of angular velocities $\omega_{1}$ covers only one decade between $\left(8.7 \times 10^{4}\right.$ and $\left.0.63 \times 10^{4}\right) \mathrm{rad} \cdot \mathrm{s}^{-1}$, as detailed above. Since both quadrupolar and heteronuclear dipolar relaxation mechanisms have nearly the same order of magnitude (see Section III.2), the experimental conditions are adequate to successfully investigate the whole dynamical range displayed in Figure 10

\section{Figure 10 and 11}

Figure 11 illustrates the free induction decays typically recorded for both $T_{1 \rho}$ and $T_{2 \rho}$ spin-locking relaxation measurements in addition with their Fourier Transforms, better exhibiting the frequency domain covered by these spin-locking relaxation measurements. Both set of figures also display the results of numerical modeling of the time evolution of the coherences (see Eqs S14 and S16 in the SI) during each step of the pulse sequences displayed in Figure 9. These numerical results were obtained by using the set of parameters displayed in Section III.2 and fitting a single empirical law describing the dispersion of the spectral 
densities. The resulting law, displayed in Figure 10, is used to determine the characteristic angular velocity $\omega_{c}$ below which all spectral densities are equal to their value at zero angular velocity and above which they all decrease according to the same logarithmic law. Figure 10 first illustrates the theoretical spectral density used to fit the spin-locking relaxation measurements displayed in figures 11a-h. As noted in the insert, this unique normalized spectral density (see the black line in Figure 10) is used to determine the six spectral densities monitoring the contributions from the quadrupolar and hetero nuclear dipolar couplings (see the SI). The only crucial parameter is the characteristic angular velocity (noted $\omega_{c}$ ) corresponding to transition between the low frequency plateau and the high frequency logarithmic decrease of the spectral density. In addition, Figure 10 also displays the distribution laws of the angular velocities respectively probed during the performed spinlocking relaxation measurements by the quadrupolar coupling, leading to the set of $\left(k_{1}\right)$ angular velocities and the hetero nuclear dipolar coupling, leading to the set of $\left(k_{2}, k_{3}\right)$ angular velocities. While the quadrupolar relaxation mechanism probes here angular velocities varying between $10^{5}$ and $3 \times 10^{5} \mathrm{rad} / \mathrm{s}$, the hetero nuclear dipolar relaxation mechanism covers a broad dynamical range varying between $10^{2}$ and $2 \times 10^{5} \mathrm{rad} / \mathrm{s}$. Since the characteristic angular velocity is localized in the middle of that probed dynamical range, we may be quite confident on the reliability of the extracted parameter $\left(\omega_{c}\right)$. The evaluation of the average residence time of the water molecules confined within the clay interlayer $\left(\tau_{c}=1 / \omega_{c}\right)$ is the main purpose of that NMR study.

As displayed in Figure 11, fair agreement with experimental data is obtained by setting the characteristic angular velocity $\omega_{c}$ equal to $10^{4} \mathrm{rad} \mathrm{s}^{-1}$ (see Figure 10). Such procedure was already used successfully to analyze ${ }^{2} \mathrm{H}$ spin-locking relaxation measurements of heavy water 
confined within various clay sediments ${ }^{25-29}$. As shown by numerical simulations of water relaxation induced by diffusion within clay interlamellar space ${ }^{27}$, the characteristic time $\tau_{c}$ defined by the inverse of the characteristic angular velocity $\tau_{c}=1 / \omega_{c}$ corresponds to the average residence time of the confined water molecules. It may be used to evaluate the order of magnitude of the self-diffusion coefficient of the confined water molecules, with $\tau_{c}=10^{-4} \mathrm{~s}$, according to the relationship:

$$
D \approx \frac{L^{2}}{2 \tau_{c}}=\frac{(3 \pm 1)^{2} \times 10^{-14} \mathrm{~m}^{2}}{2 \times 10^{-4} \mathrm{~s}}=(6 \pm 3) \times 10^{-10} \mathrm{~m}^{2} \cdot \mathrm{s}^{-1}
$$

where $L$ is the average size of the clay platelets (see Section II.1). Whatever the dimensionality of the diffusion space, the self-diffusion coefficient is defined by the ratio between the mean squared displacement and the diffusion time, leading to Eq. 3. For 2D diffusion one would rather expect:

$$
D=\lim _{\tau \rightarrow \infty} \frac{(d x)^{2}+(d y)^{2}}{4 d \tau}
$$

However, Eq. 3 simply formulates the derivation of the order of magnitude of the selfdiffusion coefficient, in agreement with previous numerical simulations of Brownian Dynamics $^{26}$. The resulting mobility is compatible with experimental data obtained by QENS $^{13,20,48,49}$ for water molecules confined within clay sediments under equivalent conditions. The water mobility of the confined water molecules evaluated by Equation 3 fully matches the radial component of the water self-diffusion tensor evaluated by MD simulations $\left(D \approx(6.6 \pm 0.5) \times 10^{-10} \mathrm{~m}^{2} / \mathrm{s}\right)$. 
This water mobility determined by ${ }^{2} \mathrm{H}$ NMR spin-locking relaxometry is at least ten times larger than the cesium mobility previously evaluated by ${ }^{133} \mathrm{Cs}$ NMR spin-locking relaxometry ${ }^{30}$ within the same sediment of fluoro-hectorite. Such a large increase of water mobility by reference to ionic mobility is not surprising because the interaction of water dipole with the charged interlayer is significantly smaller than the strong electrostatic coupling between negatively charged clay surface and the neutralizing counterions. The water mobility reported here by ${ }^{2} \mathrm{H}$ spin-locking relaxometry is twice as large as the value previously measured by $\mathrm{QENS}^{20}$ and ${ }^{2} \mathrm{H}$ spin-locking relaxometry ${ }^{28}$ within either fluorinated or hydroxylated hectorite neutralized by sodium counterions and under the same hydration condition, i.e. when confined water molecules form a single hydration layer with a characteristic period of $12.2 \AA^{33}$. That difference originates from the significant increase of the ion/water interactions after the exchange of cesium counterions by the smaller ${ }^{50}$ and more hydrophilic ${ }^{51}$ sodium cations.

\section{Conclusions}

${ }^{2} \mathrm{H}$ NMR was successfully used to quantify the influence of confinement on the structural and dynamical properties of water molecules physisorbed within dense clay sediments. ${ }^{2} \mathrm{H}$ NMR spectroscopy was first used to probe the influence of the chemical composition of the clay surface on the ordering of water molecules pertaining to the first hydration layer in the clay interlamellar space. Replacing polar hydroxides groups at the surface of clay platelets by hydrophobic fluorine atoms significantly enhances the residual quadrupolar coupling felt by the confined water molecules, quantifying the increase of water ordering at contact with the clay surface. Multi-quanta ${ }^{2} \mathrm{H}$ NMR relaxation measurements were then performed to determine the contributions of the quadrupolar and hetero-nuclear dipolar couplings responsible for the NMR relaxation of the confined water molecules. The 
high sensitivity of the time evolution of the $T_{21}^{I R}$ coherence on the residual quadrupolar coupling was also exploited to determine the distribution of the individual clay directors within the macroscopic sediment. Finally, spin-locking relaxometry measurements were used to determine the average residence time of water molecules confined within the interlamellar space of the clay platelets, leading to reliable estimate of the impact of confinement on the decrease of water mobility. This extraction of dynamical information on the mobility of confined water molecules requires the use of some theoretical formalism able to numerically describe the time evolution of the various spin states probed by the ${ }^{2} \mathrm{H}$ nuclei during each step of the pulse sequences used to perform the required NMR measurements. While this study focuses on the clay/water interface, it may be extended to other interfacial systems, including zeolitic porous networks, membranes or liquid crystals where information about the dynamical properties of confined fluids is of prime importance.

\section{Associated Content}

Supporting Information. Full details concerning the NMR relaxation theory and necessary to understand the data analysis are provided in the Supporting Information (SI).

\section{Author Information}

\section{Corresponding Authors:}

*E-mail: delville@cnrs-orleans.fr (A.D.) and porion@cnrs-orleans.fr (P.P.).

\section{ORCID:}

Patrice Porion: 0000-0003-4380-5995

\section{Notes:}

The authors declare no competing financial interest. 


\section{Acknowledgments}

The DSX360 Bruker spectrometer used for that study was purchased thanks to grants from Région Centre (France). We acknowledge the contribution from the NEEDS interdisciplinary project (MIPOR, MULTIDYN). We cordially thank Dr Joseph Breu (Institut für Anorganische Chemie der Universität Regensburg) for providing Hectorite clay sample. The authors are grateful to José C. Gomes and Santiago Braley for their help in the design and the machining of the glass cell. 


\section{References}

(1)Israelachvili, J. N. Intermolecular and Surface Forces; Academic Press: New York, 1985.

(2) Bowers, G. M.; Singer, J. W.; Bish, D. L.; Kirkpatrick, R. J. Alkali Metal and $\mathrm{H}_{2} \mathrm{O}$ Dynamics at the Smectite/Water Interface. J. Phys. Chem. C 2011, 115, 23395-23407.

(3) Henderson, D. Fundamentals of Inhomogeneous Fluids; M. Dekker: New York, 1992.

(4) Cygan, R. T.; Greathouse, J. A.; Heinz, H.; Kalinichev, A. G. Molecular Models and Simulations of Layered Materials. J. Mater. Chem. 2009, 19, 2470-2481.

(5) Jobbagy, M.; Iyi, N. Interplay of Charge Density and Relative Humidity on the Structure of Nitrate Layered Double Hydroxides. J. Phys. Chem. C 2010, 114, 18153-18158.

(6) Lee, S. S.; Fenter, P.; Park, C.; Sturchio, N. C.; Nagy, K. L. Hydrated Cation Speciation at the Muscovite (001)-Water Interface. Langmuir 2010, 26, 16647-16651.

(7) Ferrage, E.; Sakharov, B. A.; Michot, L. J.; Delville, A.; Bauer, A.; Lanson, B.; Grangeon, S.; Frapper, G.; Jiménez-Ruiz, M.; Cuello, G. J. Hydration Properties and Interlayer Organization of Water and Ions in Synthetic Na-Smectite with Tetrahedral Layer Charge. Part 2. Toward a Precise Coupling between Molecular Simulations and Diffraction Data. J. Phys. Chem. C 2011, 115, 1867-1881.

(8) Boily, J. F. Water Structure and Hydrogen Bonding at Goethite/Water Interfaces: Implications for Proton Affinities. J. Phys. Chem. C 2012, 116, 4714-4724.

(9) Ho, T. A.; Argyris, D.; Cole, D. R.; Striolo, A. Aqueous $\mathrm{NaCl}$ and CsCl Solutions Confined in Crystalline Slit-Shaped Silica Nanopores of Varying Degree of Protonation. Langmuir 2012, 28, 1256-1266.

(10) Briman, I. M.; Rébiscoul, D.; Diat, O.; Zanotti, J. M.; Jollivet, P.; Barboux, P.; Gin, S. Impact of Pore Size and Pore Surface Composition on the Dynamics of Confined Water in Highly Ordered Porous Silica. J. Phys. Chem. C 2012, 116, 7021-7028.

(11) Gates, W. P.; Bordallo, H. N.; Aldridge, L. P.; Seydel, T.; Jacobsen, H.; Marry, V.; Churchman, G. J. Neutron Time-of-Flight Quantification of Water Desorption Isotherms of Montmorillonite. J. Phys. Chem. C 2012, 116, 5558-5570.

(12) Malani, A.; Ayappa, K. G. Relaxation and Jump Dynamics of Water at the Mica Interface. J. Chem. Phys. 2012, 136, 194701.

(13) Michot, L. J.; Ferrage, E.; Jiménez-Ruiz, M.; Boehm, M.; Delville, A. Anisotropic Features of Water and Ion Dynamics in Synthetic Na- and Ca-Smectites with Tetrahedral Layer Charge. A Combined Quasi-Elastic Neutron-Scattering and Molecular Dynamics Simulations Study. J. Phys. Chem. C 2012, 116, 16619-16633.

(14) Handbook of Clay Science; $1^{\text {st }}$ ed.; Bergaya, F.; Theng, B. K. G.; Lagaly, G., Eds.; Elsevier: Amsterdam, 2006; Vol. 1.

(15) Jiménez-Ruiz, M.; Ferrage, E.; Delville, A.; Michot, L. J. Anisotropy on the Collective Dynamics of Water Confined in Swelling Clay Minerals. J. Phys. Chem. A 2012, 116, 2379-2387.

(16) Cygan, R. T.; Daemen, L. L.; Ilgen, A. G.; Krumhansl, J. L.; Nenoff, T. M. Inelastic Neutron Scattering and Molecular Simulation of the Dynamics of Interlayer Water in Smectite Clay Minerals. J. Phys. Chem. C 2015, 119, 28005-28019. 
(17) Michot, L. J.; Ferrage, E.; Delville, A.; Jiménez-Ruiz, M. Influence of Layer Charge, Hydration State and Cation Nature on the Collective Dynamics of Interlayer Water in Synthetic Swelling Clay Minerals. Appl. Clay Sci. 2016, 119, 375-384.

(18) Jiménez-Ruiz, M.; Ferrage, E.; Blanchard, M.; Fernandez-Castanon, J.; Delville, A.; Johnson, M. R.; Michot, L. J. Combination of Inelastic Neutron Scattering Experiments and $\mathrm{Ab}$ Initio Quantum Calculations for the Study of the Hydration Properties of Oriented Saponites. J. Phys. Chem. C 2017, 121, 5029-5040.

(19) Michot, L. J.; Delville, A.; Humbert, B.; Plazanet, M.; Levitz, P. Diffusion of Water in a Synthetic Clay with Tetrahedral Charges by Combined Neutron Time-of-Flight Measurements and Molecular Dynamics Simulations. J. Phys. Chem. C 2007, 111, $9818-$ 9831.

(20) Marry, V.; Dubois, E.; Malikova, N.; Durand-Vidal, S.; Longeville, S.; Breu, J. Water Dynamics in Hectorite Clays: Influence of Temperature Studied by Coupling Neutron Spin Echo and Molecular Dynamics. Environ. Sci. Technol. 2011, 45, 2850-2855.

(21) Callaghan, P. T. Principles of Nuclear Magnetic Resonance Microscopy; Clarendon Press: Oxford, 1991.

(22) Kimmich, R.; Fatkullin, N. Self-Diffusion Studies by Intra- and Inter-Molecular SpinLattice Relaxometry Using Field-Cycling: Liquids, Plastic Crystals, Porous Media, and Polymer Segments. Prog. Nucl. Magn. Reson. Spectrosc. 2017, 101, 18-50.

(23) Korb, J. P.; Delville, A.; Xu, S.; Demeulenaere, G.; Costa, P.; Jonas, J. Relative Role of Surface Interactions and Topological Effects in Nuclear Magnetic Resonance of Confined Liquids. J. Chem. Phys. 1994, 101, 7074-7081.

(24) Korb, J.-P. Multiscale Nuclear Magnetic Relaxation Dispersion of Complex Liquids in Bulk and Confinement. Prog. Nucl. Magn. Reson. Spectrosc. 2018, 104, 12-55.

(25) Porion, P.; Michot, L. J.; Faugère, A. M.; Delville, A. Structural and Dynamical Properties of the Water Molecules Confined in Dense Clay Sediments: A Study Combining ${ }^{2} \mathrm{H}$ NMR Spectroscopy and Multiscale Numerical Modeling. J. Phys. Chem. C 2007, 111, 5441-5453.

(26) Porion, P.; Michot, L. J.; Faugère, A. M.; Delville, A. Influence of Confinement on the Long-Range Mobility of Water Molecules within Clay Aggregates: A ${ }^{2} \mathrm{H}$ NMR Analysis Using Spin-Locking Relaxation Rates. J. Phys. Chem. C 2007, 111, 13117-13128.

(27) Porion, P.; Michot, L. J.; Warmont, F.; Faugère, A. M.; Delville, A. Long-Time Dynamics of Confined Water Molecules Probed by ${ }^{2} \mathrm{H}$ NMR Multiquanta Relaxometry: An Application to Dense Clay Sediments. J. Phys. Chem. C 2012, 116, 17682-17697.

(28) Porion, P.; Faugère, A. M.; Delville, A. Multiscale Water Dynamics within Dense Clay Sediments Probed by ${ }^{2} \mathrm{H}$ Multiquanta NMR Relaxometry and Two-Time Stimulated Echo NMR Spectroscopy. J. Phys. Chem. C 2013, 117, $26119-26134$.

(29) Porion, P.; Faugère, A. M.; Delville, A. Structural and Dynamical Properties of Water Molecules Confined within Clay Sediments Probed by Deuterium NMR Spectroscopy, Multiquanta Relaxometry, and Two-Time Stimulated Echo Attenuation. J. Phys. Chem. C 2014, 118, 20429-20444.

(30) Porion, P.; Warmont, F.; Faugère, A. M.; Rollet, A.-L.; Dubois, E.; Marry, V.; Michot, L. J.; Delville, A. ${ }^{133}$ Cs Nuclear Magnetic Resonance Relaxometry as a Probe of the 
Mobility of Cesium Cations Confined within Dense Clay Sediments. J. Phys. Chem. C 2015, 119, 15360-15372.

(31) Kimmich, R.; Anoardo, E. Field-Cycling NMR Relaxometry. Prog. Nucl. Magn. Reson. Spectrosc. 2004, 44, 257-320.

(32) Dazas, B.; Lanson, B.; Breu, J.; Robert, J.-L.; Pelletier, M.; Ferrage, E. Smectite Fluorination and Its Impact on Interlayer Water Content and Structure: A Way to Fine Tune the Hydrophilicity of Clay Surfaces? Microporous Mesoporous Mater. 2013, 181, 233-247.

(33) Dazas, B.; Lanson, B.; Delville, A.; Robert, J.-L.; Komarneni, S.; Michot, L. J.; Ferrage, E. Influence of Tetrahedral Layer Charge on the Organization of Interlayer Water and Ions in Synthetic Na-Saturated Smectites. J. Phys. Chem. C 2015, 119, 4158-4172.

(34) Breu, J.; Seidl, W.; Stoll, A. J.; Lange, K. G.; Probst, T. U. Charge Homogeneity in Synthetic Fluorohectorite. Chem. Mater. 2001, 13, 4213-4220.

(35) Neilson, G. W.; Enderby, J. E. Chapter 7. Neutron and X-Ray Diffraction Studies of Concentrated Aqueous Electrolyte Solutions. Annu. Rep. Prog. Chem., Sect. C, Phys. Chem. 1979, 76, 185-220.

(36) Müller, N.; Bodenhausen, G.; Ernst, R. R. Relaxation-Induced Violations of Coherence Transfer Selection Rules in Nuclear Magnetic Resonance. J. Magn. Reson. 1987, 75, 297-334.

(37) van der Maarel, J. R. C. The Relaxation Dynamics of Spin I=1 Nuclei with a Static Quadrupolar Coupling and a Radio-Frequency Field. J. Chem. Phys. 1993, 99, 56465653.

(38) Plimpton, S. Fast Parallel Algorithms for Short-Range Molecular Dynamics. J. Comput. Phys. 1995, 117, 1-19.

(39) Cygan, R. T.; Liang, J. J.; Kalinichev, A. G. Molecular Models of Hydroxide, Oxyhydroxide, and Clay Phases and the Development of a General Force Field. J. Phys. Chem. B 2004, 108, 1255-1266.

(40) Abascal, J. L. F.; Vega, C. A General Purpose Model for the Condensed Phases of Water: TIP4P/2005. J. Chem. Phys. 2005, 123, 234505.

(41) Reddy, U. V.; Bowers, G. M.; Loganathan, N.; Bowden, M.; Yazaydin, A. O.; Kirkpatrick, R. J. Water Structure and Dynamics in Smectites: X-Ray Diffraction and ${ }^{2} \mathrm{H}$ NMR Spectroscopy of $\mathrm{Mg}-, \mathrm{Ca}-, \mathrm{Sr}-, \mathrm{Na}-, \mathrm{Cs}-$, and $\mathrm{Pb}-$ Hectorite. J. Phys. Chem. C 2016, 120, 8863-8876.

(42) Woessner, D. E. Nuclear Transfer Effects in Nuclear Magnetic Resonance Pulse Experiments. J. Chem. Phys. 1961, 35, 41-48.

(43) Fukushima, E.; Roeder, S. B. W. Experimental Pulse NMR: A Nuts and Bolts Approach; Addison-Wesley: Reading, MA, 1981.

(44) Petit, D.; Korb, J. P.; Delville, A.; Grandjean, J.; Laszlo, P. Theory of Nuclear Spin Relaxation in Heterogeneous Media and Application to the Cross Correlation between Quadrupolar and Dipolar Fluctuations of Deuterons in Clay Gels. J. Magn. Reson. 1992, 96, 252-279. 
(45) Barbara, T. M.; Vold, R. R.; Vold, R. L. A Determination of Individual Spectral Densities in a Smectic Liquid-Crystal from Angle Dependent Nuclear Spin Relaxation Measurements. J. Chem. Phys. 1983, 79, 6338-6340.

(46) Hwang, D. W.; Jhao, W.-J.; Hwang, L.-P. ${ }^{2} \mathrm{H}^{\mathrm{T}} \mathrm{T}_{2 \rho}$ Relaxation Dynamics and DoubleQuantum Filtered NMR Studies. J. Magn. Reson. 2005, 172, 214-221.

(47) Blicharski, J. S. Nuclear Magnetic Relaxation in Rotating Frame. Acta Phys. Pol. 1972, A41, 223-236.

(48) Malikova, N.; Cadène, A.; Dubois, E.; Marry, V.; Durand-Vidal, S.; Turq, P.; Breu, J.; Longeville, S.; Zanotti, J. M. Water Diffusion in a Synthetic Hectorite Clay Studied by Quasi-Elastic Neutron Scattering. J. Phys. Chem. C 2007, 111, 17603-17611.

(49) Marry, V.; Dubois, E.; Malikova, N.; Breu, J.; Haussler, W. Anisotropy of Water Dynamics in Clays: Insights from Molecular Simulations for Experimental QENS Analysis. J. Phys. Chem. C 2013, 117, 15106-15115.

(50) Enderby, J. E.; Neilson, G. W. The Structure of Electrolyte Solutions. Rep. Prog. Phys. 1981, 44, 593-653.

(51) Rashin, A. A.; Honig, B. Reevaluation of the Born Model of Ion Hydration. J. Phys. Chem. 1985, 89, 5588-5593. 
p. 26

Figure 1

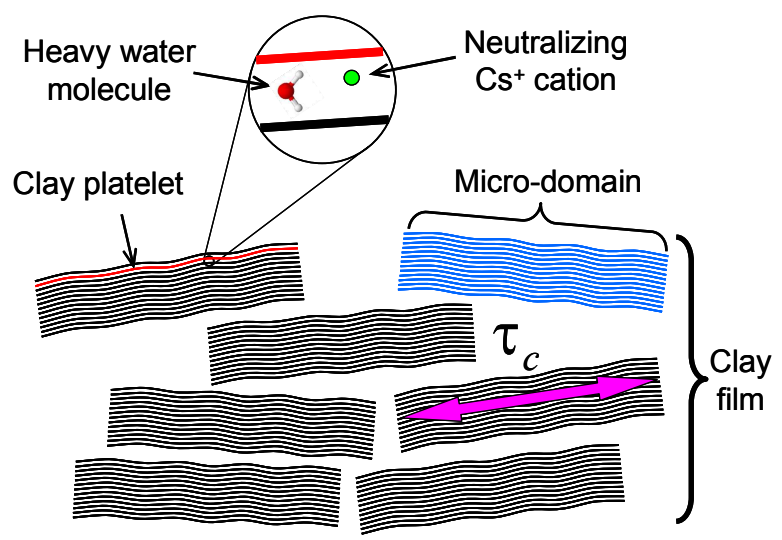

Figure 1. Schematic view of the multi-scale organization of the clay sample within the selfsupporting film. 
Figure 2
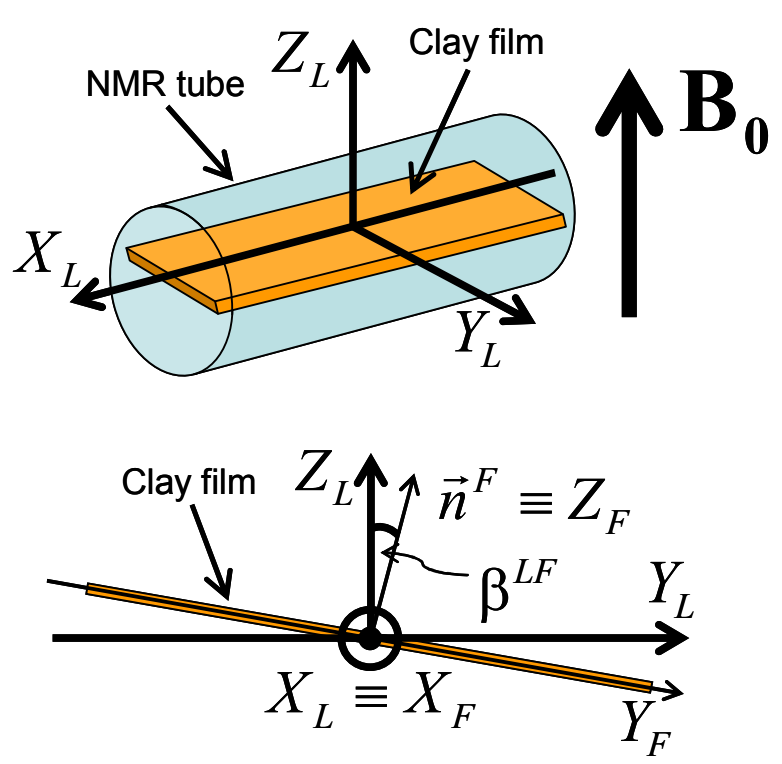

Figure 2. Schematic view of the film orientation within the NMR tube used to insert the clay sample into the detection coil within the static magnetic field $\mathbf{B}_{\mathbf{0}}$ (see text). 
p. 28

Figure 3

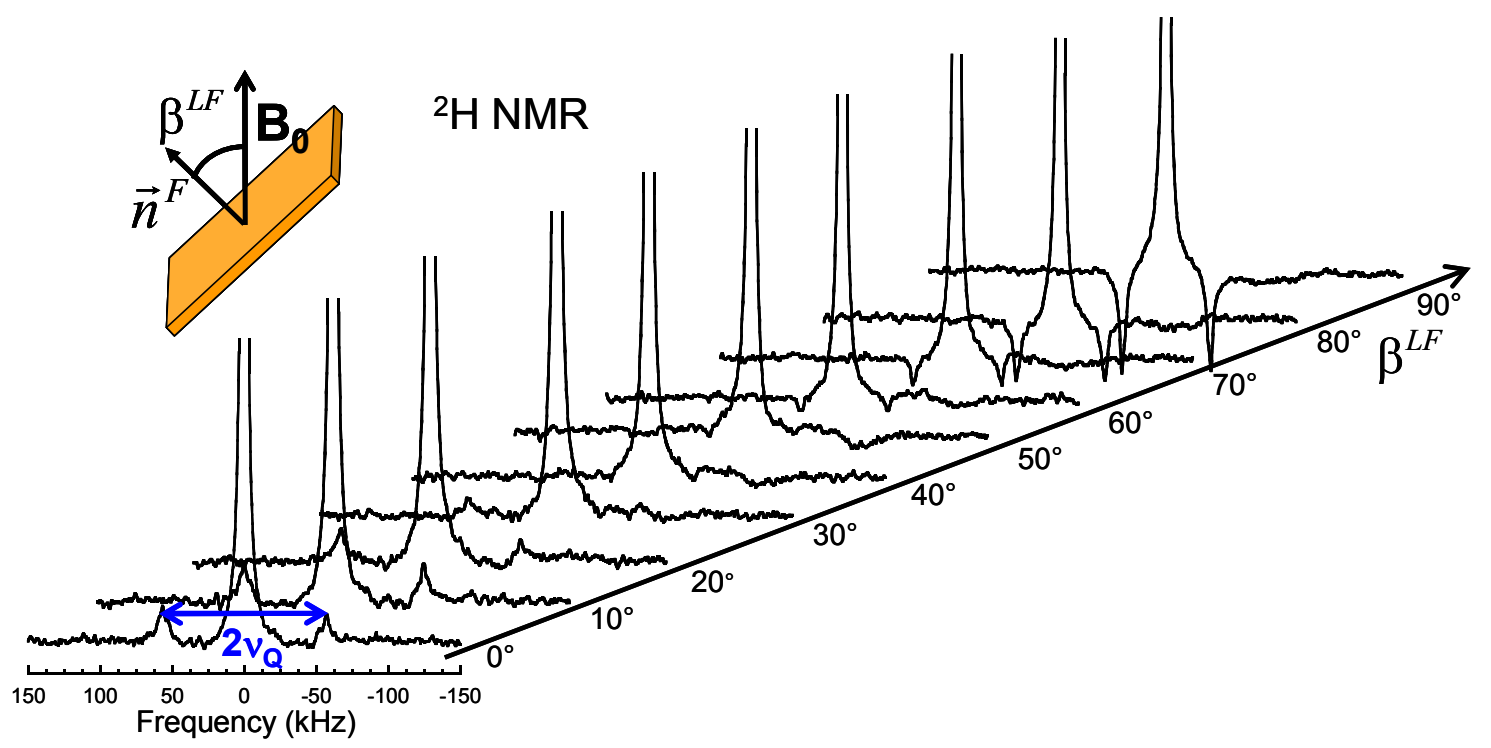

Figure 3. Variation of the ${ }^{2} \mathrm{H}$ NMR spectra of confined and bulk water molecules as a function of the orientation $\beta^{L F}$ of the film director $\vec{n}^{F}$ into the static magnetic field $\mathbf{B}_{0}$ (see text and Figure 2). 


\section{Figure 4}
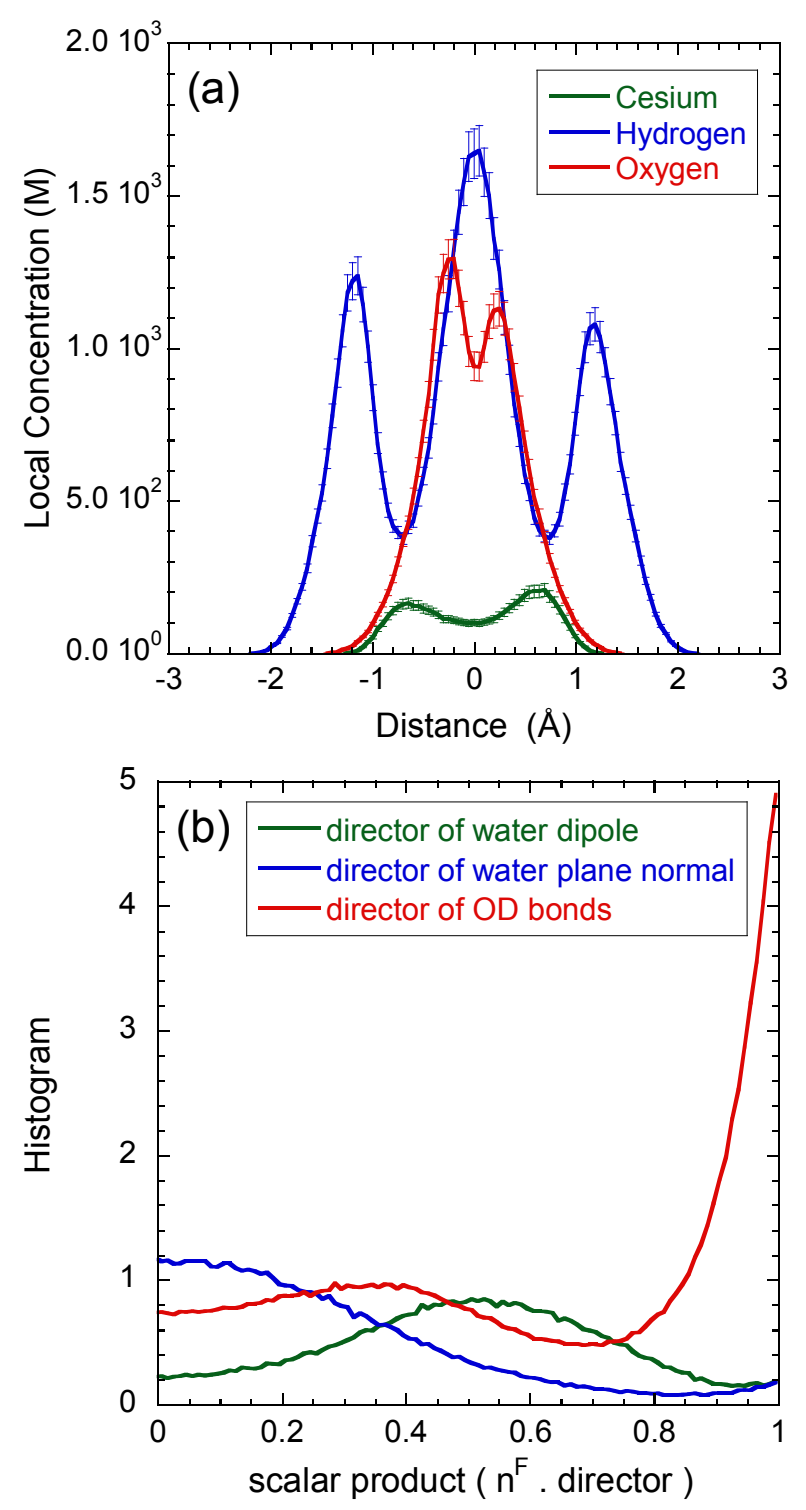

Figure 4. (a) Concentrations profiles of the neutralizing cesium cation in addition to the hydrogen and oxygen atoms of the confined water molecules. The plots are centered on the equatorial plane located at the middle of the interlamellar space. (b) Analysis of the orientation of the confined water molecules based on the distributions, by reference to the normal of the clay platelets, of three different directors attached to the water molecules and describing respectively the water dipole, the normal to the $D O D$ plane and the $O D$ director (see text). 


\section{Figure 5}

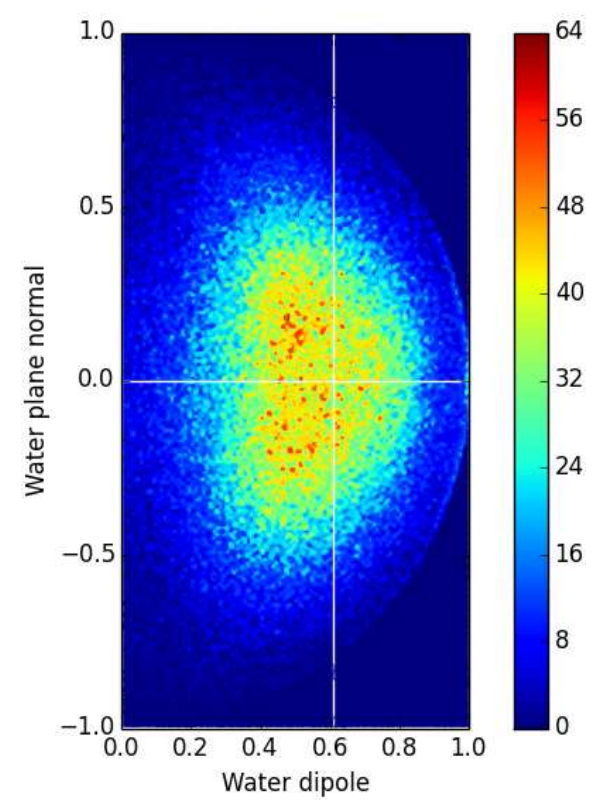

Figure 5. 3D plot illustrating the ordering of the confined water molecules characterized by the simultaneous distribution of the orientation of the water dipole and the normal to the $D O D$ plane by reference to the normal of the clay platelet. Details concerning the horizontal and vertical white reference lines are given in the text. 


\section{Figure 6}

Figure 6. Pulse sequences and coherences transfer pathways used to measure (a) the relaxation rate $R_{10}=1 / T_{1}$ of the coherence $T_{10}^{I R}$ by a saturation-recovery pulse sequence followed by a double-quanta filtering. The multiquanta relaxation rates (b) $R_{21}$ and (c) $R_{22}$ of the $T_{21}^{I R}$ and $T_{22}^{I R}$ coherences are respectively measured using pre-saturation pulses sequence and double-quanta filtering (see text). In all sequences, the evolution time $\tau$ is varied to sample the relaxation rates. The delay $\varepsilon$ inserted between the two filtering pulses, for (a) and (b) sequences, is set equal to its minimum values (i.e. $5 \mu \mathrm{s}$ ). Finally for (b) and (c) sequences, the delay $\delta_{o p t}$ is set to $40 \mathrm{~ms}$ in order to recover the maximum magnetization from the confined water molecules and simultaneously minimizes the magnetization from the bulk water by exploiting the difference of their longitudinal relaxation rates $R_{10}$ (see text). 


\section{Figure 7}
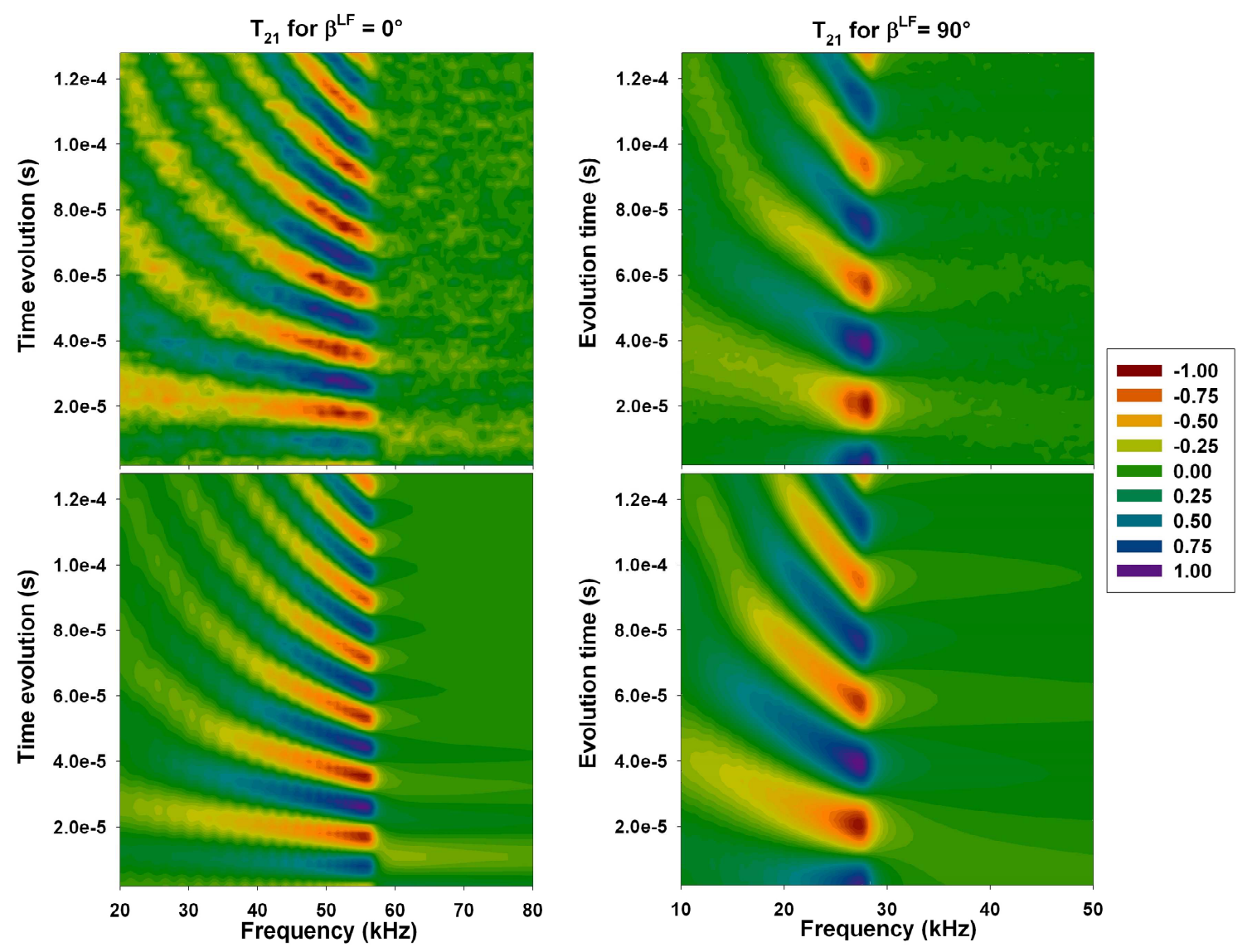

Figure 7. 3D plots illustrated the comparison between the experimental (top) and simulated (bottom) time evolution of the $T_{21}^{I R}$ coherence performed for parallel, $\beta^{L F}=0^{\circ}$ (left), and perpendicular, $\beta^{L F}=90^{\circ}$ (right), orientations of the clay directors $\vec{n}^{F}$ into the static magnetic field $\mathbf{B}_{\mathbf{0}}$ (see text). 


\section{Figure 8}
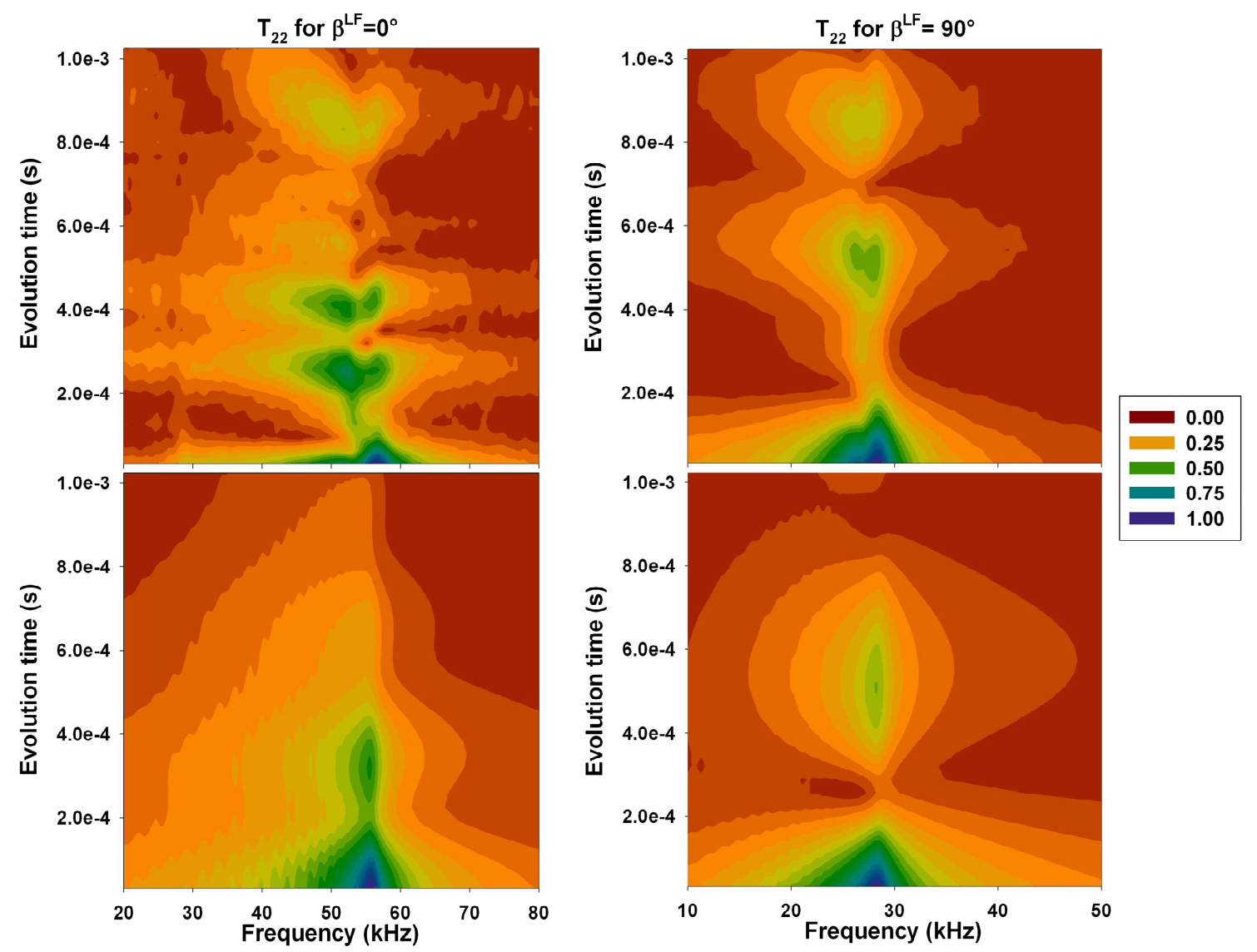

Figure 8. . 3D plots illustrated the comparison between the experimental (top) and simulated (bottom) time evolution of the $T_{22}^{I R}$ coherence performed for parallel, $\beta^{L F}=0^{\circ}$ (left), and perpendicular, $\beta^{L F}=90^{\circ}$ (right), orientations of the clay directors $\vec{n}^{F}$ into the static magnetic field $\mathbf{B}_{\mathbf{0}}$ (see text). 
p. 34

Figure 9
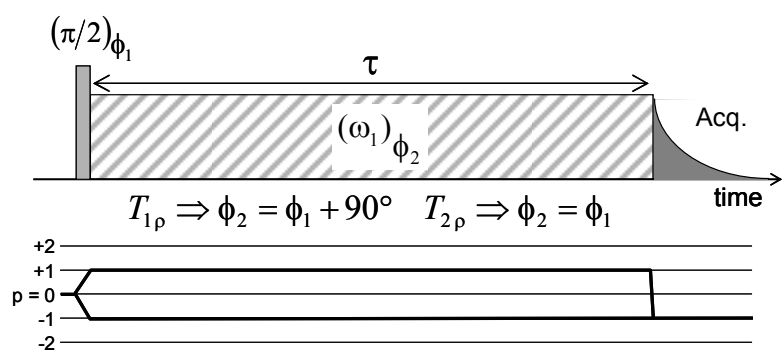

Figure 9. Pulse sequences and coherence pathways used to perform $T_{1 \rho}$ and $T_{2 \rho}$ spin-locking relaxometry measurements. 
Figure 10

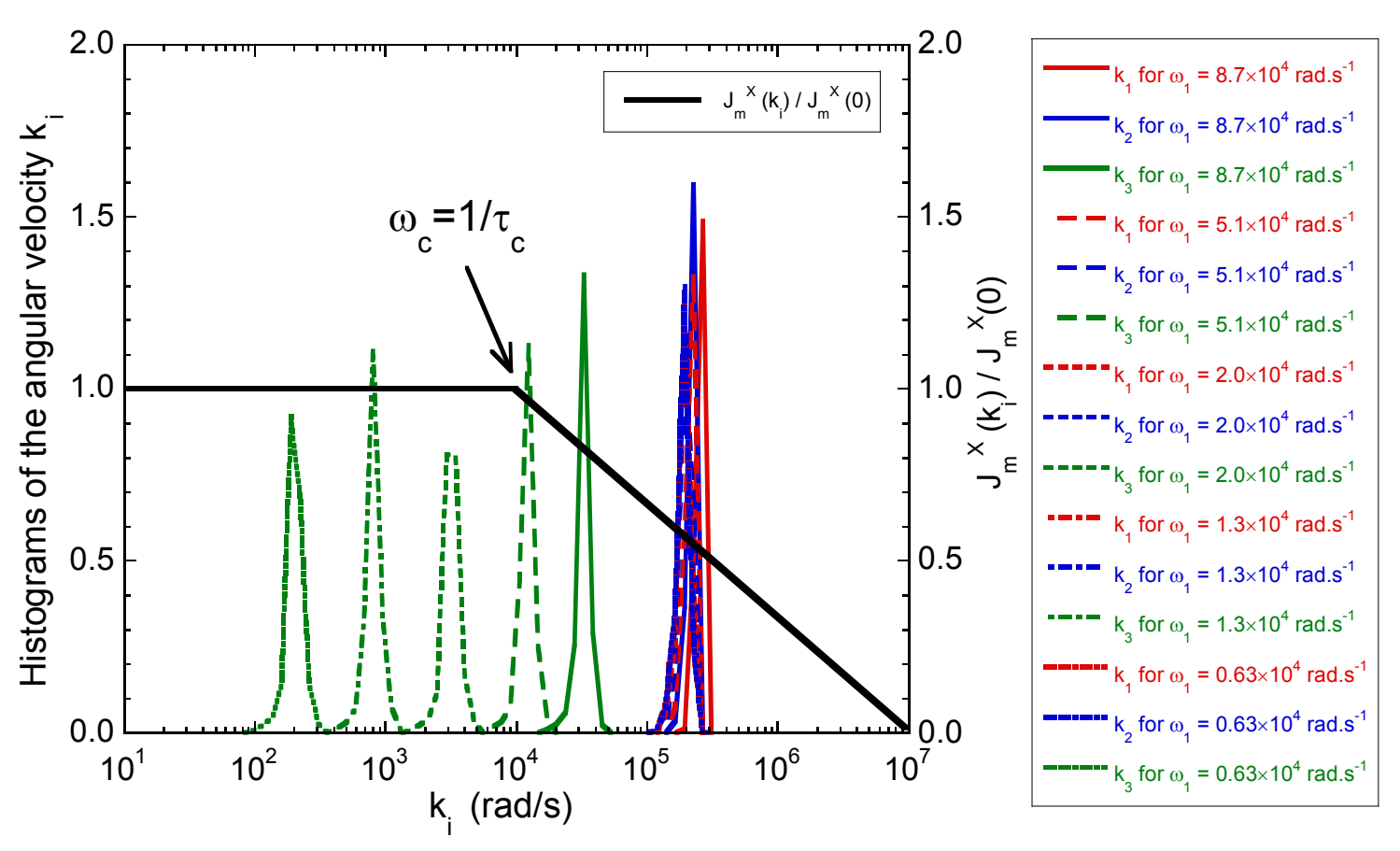

Figure 10. Distribution of the complete sets of resonance angular velocities $k_{i} i \in\{1,2,3\}$, probed by $T_{1 \rho}$ and $T_{2 \rho}$ spin-locking relaxometry measurements performed at 5 different irradiating fields $\omega_{1}$, in addition to the fitted dispersion curve exhibiting the characteristic angular velocity $\omega_{c}$ (see text). 
Figure 11
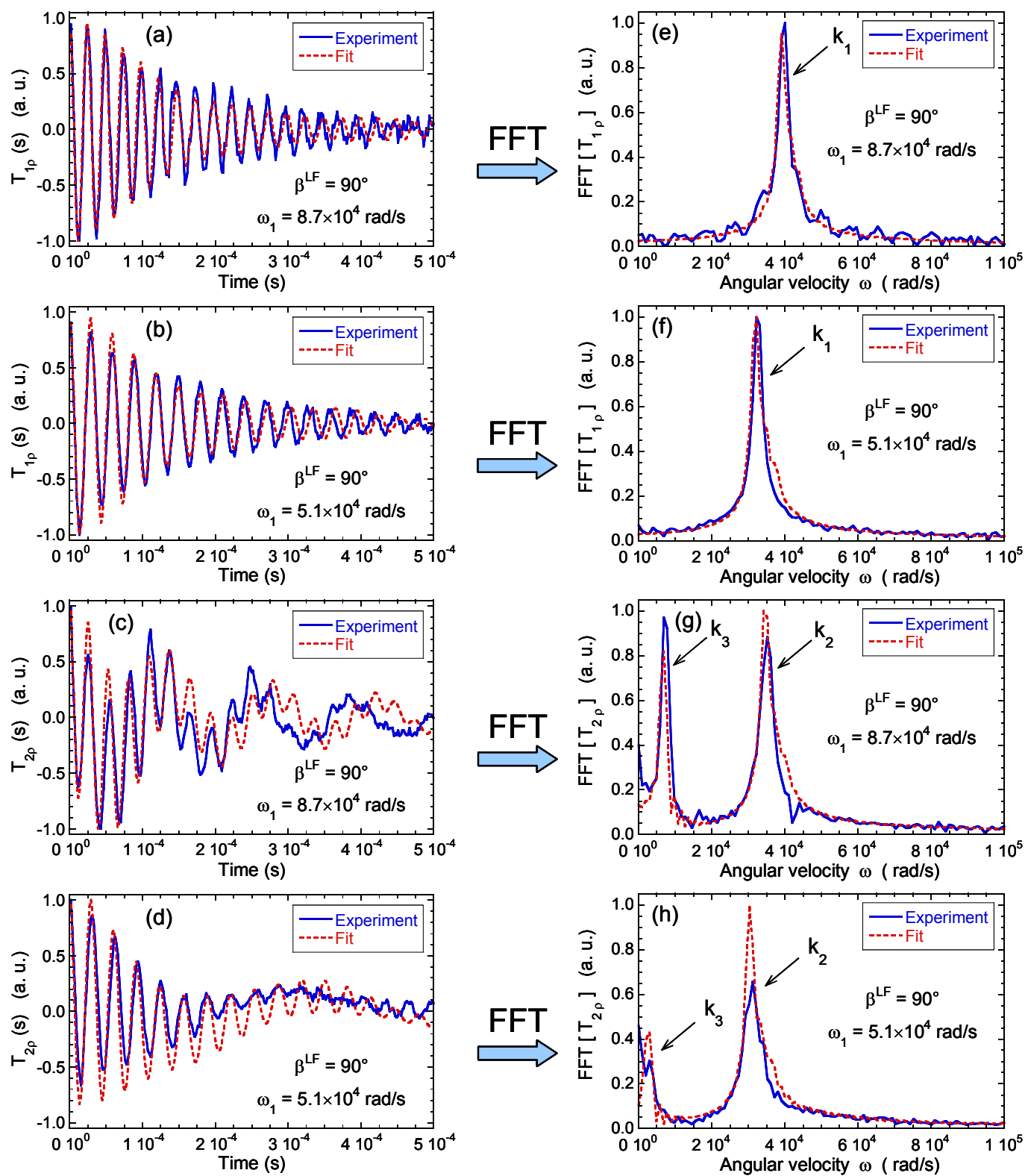

Figure 11. Comparison between measured and fitted $T_{1 \rho}$ (top, a-b and e-f) and $T_{2 \rho}$ (bottom, c-d and g-h) spin-locking relaxation rates measured at two angular velocities, $\omega_{1}=8.7 \times 10^{4} \mathrm{rad} / \mathrm{s}$ and $5.1 \times 10^{4} \mathrm{rad} / \mathrm{s}$ for $\beta^{L F}=90^{\circ}$ (see text). The time evolutions of the magnetization (left, a-d) are displayed in addition to their Fourier transform (right, e-h). 
Figure for Table of Contents (TOC) graphic

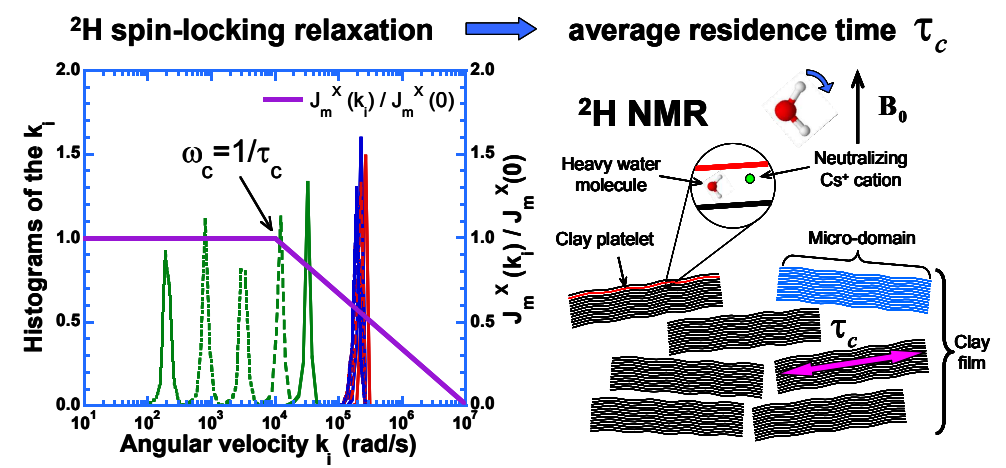

